\title{
EPIDEMIOLOGY, CLINICAL AND CYTOLOGICAL FEATURES OF LYMPHOMA IN BOXER DOGS
}

\author{
Urszula JANKOWSKA $^{1}$, Dariusz JAGIELSKI ${ }^{1}$, Michał CZOPOWICZ ${ }^{2}$ and \\ Rafał SAPIERZYŃSKI ${ }^{3 *}$ \\ ${ }^{1}$ Białobrzeska Veterinary Surgery, Warsaw, Poland; ${ }^{2}$ Laboratory of Veterinary \\ Epidemiology and Economics, Faculty of Veterinary Medicine, Warsaw University \\ of Life Sciences (SGGW), Warsaw, Poland; ${ }^{3}$ Division of Animal Pathomorphology, \\ Department of Pathology and Veterinary Diagnostics and Faculty of Veterinary \\ Medicine, Warsaw University of Life Sciences (SGGW), Nowoursynowska str. 166, \\ 02-787 Warsaw, Poland
}

(Received 3 September 2018; accepted 20 February 2019)

The aim of this study was to evaluate the epidemiology, clinical and laboratory characteristics of canine lymphomas as well as some aspects of treatment outcomes. The study was conducted on Boxer dogs with lymphoma diagnosed by cytology and immunocytochemistry (CD3 and CD79 alpha). During the study period, lymphoma was diagnosed in 63 Boxers; $86.8 \%$ were T-cell (based on the Kiel classification: small clear cell lymphoma, pleomorphic small cell lymphoma, pleomorphic mixed T-cell lymphoma, pleomorphic large T-cell lymphoma, lymphoblastic lymphoma/acute lymphoblastic leukaemia) and $13.2 \%$ were B-cell lymphomas (according to the Kiel classification: B-cell chronic lymphocytic leukaemia, centroblastic/centroblastic polymorphic lymphoma). Overall survival (OS) was significantly longer in dogs with low-grade than with high-grade lymphoma (median OS of 6.8 and 4.7 months, respectively; $\mathrm{P}=0.024$ ). OS was not influenced by WHO clinical stage, WHO clinical substage, presence of splenomegaly, early administration of glucocorticoids or the time from the first presentation to the beginning of chemotherapy. There are no significant differences in clinical and laboratory parameters between low-grade and high-grade lymphomas. Boxer dogs are predisposed to T-cell lymphoma, with a predominance of highgrade tumour, especially pleomorphic, mixed small and large T-cell subtype. It is possible that Boxer dogs may respond less favourably to chemotherapy than patients of other breeds.

Key words: Boxer, cytology, dog, lymphoma, T-cell lymphoma

Breed predilections for specific lymphoma subtypes are well characterised in the veterinary literature; however, descriptions of larger groups of affected dogs of one breed are uncommon (Lurie et al., 2004; Lurie et al., 2008; Sutter

*Corresponding author; sapieh@wp.pl; Phone: 0048 (22) 593-6135; Fax: 0048 (22) 593-6152 
and Ostrander, 2004; Modiano et al., 2005; Dobson, 2013). Even though the aetiology of most neoplasms appears to be multifactorial, the high level of genetic homogeneity within dog breeds is presumably responsible, to some extent, for predisposition to specific tumours (Sutter and Ostrander, 2004; Modiano et al., 2005; Dobson, 2013). The prevalence of T-cell lymphoproliferative disease is the highest in Spitz breeds (Akita, Basenji, Siberian Husky, Alaskan Malamute, Chinese Shar-Pei and wolves) and in the Shih Tzu group (Shih Tzu, Lhasa Apso, and other Asian 'lap' dogs; Modiano et al., 2005). Boxers show an increased risk of developing T-cell tumours, since as many as 55 to $85 \%$ of Boxers with lymphoma have tumours of T-cell immunophenotype (Lurie et al., 2004; Modiano et al., 2005; Lurie et al., 2008; Pastor et al., 2009; Jankowska et al., 2015). The limited genetic diversity within dog breeds facilitates genetic linkage or association studies on relatively small populations as compared to humans (Modiano et al., 2005; Cadieu and Ostrander, 2007; Olson, 2007; Shearin and Ostrander, 2010). The studies regarding predilection for different types of tumours in the canine population may be beneficial for elucidating the aetiology and pathogenesis of human neoplasms.

This strong breed association with different subtypes of lymphoma may at least partly explain differences in clinical presentation, response to chemotherapy and survival time in certain dog breeds (Garret et al., 2002). Dogs with some subtypes of T-cell lymphoma are at a significantly higher risk of poorer response to therapy as well as relapse and early death following therapy compared to those with B-cell lymphoma (Ruslander et al., 1997; Ponce et al., 2003). The aim of this study was to evaluate the epidemiological, clinical and laboratory characteristics of lymphoma-affected Boxer dogs, including some aspects of treatment outcomes.

\section{Materials and methods}

\section{Study population and collection of cases}

The study was conducted on canine patients in 2009-2015. Lymphoma was diagnosed in 63 Boxer dogs, but full data were available only for 29 of them. The rest of the dogs without full data available $(n=34)$ were included in the epidemiological and cytological analyses. In the study period, all cases diagnosed cytologically as canine lymphomas were provisionally collected and data on age, breed, and sex were recorded. In every Boxer dog with a diagnosis of lymphoma, additionally the results of full clinical examination, abdominal ultrasonography, complete blood count and biochemical parameters including total calcium, albumins, total protein, alanine aminotransferase (ALT), aspartate aminotransferase (AST), alkaline phosphatase (AP), total bilirubin, creatinine and urea were measured in laboratories with systems of daily internal and periodic external quality control ('VetLab' and 'LabWet' in Warsaw, Poland; using Dia- 
tron Abacus Vet5, Cormay Mythic 18, MetroLab 2300 PLUS, Siemens RapiDChem 744, Siemens Immulite 2000 XPi, Siemens DPC Immulite One, ERBA 640 XL and Mindray BS-800 devices). In addition, information was collected about the treatment scheme and its results, if available. Smears for cytological examination were obtained by fine-needle aspiration or non-aspiration biopsy of enlarged lymph nodes (in cases of systemic lymphadenomegaly samples were collected from at least two enlarged lymph nodes and at least three samples from each examined lymph node were taken) and/or from abnormal masses located on the skin (in two cases) and in body cavities (mediastinal tumours - 5 dogs and abdominal cavity tumours - one patient), bone marrow, if leukocytosis was observed in peripheral blood smears (one each from group with T-cell and B-cell lymphoma) and fluids from serosal cavities (sediment fluids from the pleural cavity were taken from two patients). Samples were also collected from the spleen or liver if splenomegaly or hepatomegaly were detected (in a total of 6 and 25 cases, respectively). If necessary, the sampling was performed in local anaesthesia or sedation/narcosis using topical lignocaine (Lignocainum hydrochloricum 2\%; Warszawskie Zakłady Farmaceutyczne Polfa, Poland) and, for general anaesthesia, butorphanol (Butomidor $10 \mathrm{mg} / \mathrm{ml}$; Richter Pharma AG, Austria), dexmedetomidine (Dexdomitor $0.5 \mathrm{mg} / \mathrm{ml}$; Orion Pharma, Finland), ketamine (Bioketan $100 \mathrm{mg} / \mathrm{ml}$; Vetoquinol Biowet Puławy, Poland) or propofol (PropofolLipuro $10 \mathrm{mg} / \mathrm{ml}$; B. Braun, Germany). Finally, all Boxer dogs, in which the cytomorphological diagnosis of lymphoma was made and its subtype was recognised unequivocally twice by the same clinical cytologist (the period between first examination and re-examination of the slides by the cytologist was between 1 and 2 months), were enrolled in the study. Moreover, to confirm the diagnosis of lymphoma subtype, immunocytochemistry was performed if a sufficient number of slides were available. For 29 cases the history of treatment was documented: in 9 Boxer dogs with low-grade lymphoma and 20 with high-grade lymphoma. The procedure was standardised to 5 groups: Group I: without treatment ( $\mathrm{n}=$ 8; 2 dogs with low-grade and 6 with high-grade lymphomas); Group II: only prednisolone ( $\mathrm{n}=9 ; 4$ cases with low-grade and 5 with high-grade lymphoma); Group III: COP-based ( $\mathrm{n}=2$; only low-grade lymphomas); Group IV: CHOPbased $(n=9)$; Group V: cyclophosphamide orally $(n=1)$. In 9 patients receiving treatment according to the CHOP-based protocol changes were introduced concerning the exclusion of Asparaginase and/or Lomustine - only the base of CHOP: $\mathrm{n}=3$; CHOP with Asparaginase: $\mathrm{n}=1$; CHOP with Lomustine: $\mathrm{n}=3$; $\mathrm{CHOP}$ with Asparaginase and Lomustine: $\mathrm{n}=2$. The modifications resulted from the course of the disease and the possibilities of dog owners. The administration of glucocorticoids before the start of chemotherapy was reported by $34 \%$ of dog owners $(\mathrm{n}=10 ; 7$ of high-grade lymphoma). The therapy lasted 38 days on average. 


\section{Slide preparation and immunocytochemistry}

For routine examination at least 3 smears of aspirates were dried, fixed in $70 \%$ methanol, stained with Giemsa solution and examined by light microscopy. For immunocytochemical assays, smears from each dog were dried, fixed in acetone at $4{ }^{\circ} \mathrm{C}$ for $5-10 \mathrm{~min}$, and stained immediately or stored at $-20{ }^{\circ} \mathrm{C}$. Immunocytochemical staining was performed according to Caniatti et al. (1996) and Sapierzyński (2010) using commercially available antibodies (Dako ${ }^{\circledR}$, Glostrup, Denmark) for the pan T-lymphocyte marker CD3 (Polyclonal Rabbit AntiHuman) and B-cell antigen receptor complex CD79 $\alpha$ (Monoclonal Mouse AntiHuman). Two smears from the same case were stained using both antibodies. The expression intensity of the examined CD antigens in cytological preparations was determined in a light microscope and the result was considered positive if at least $80 \%$ of the lymphoid cells showed a strong cytoplasmic reaction (i.e., T cell immunophenotype was established if at least $80 \%$ of the lymphocytes were CD3 immunopositive, and B cell immunophenotype was established if at least $80 \%$ of the lymphocytes were CD79 $\alpha$ immunopositive). The entire slide was searched, and immunopositive cells were calculated among 1000 cells using an Olympus BX41 microscope coupled to a computer equipped with CellA ${ }^{\circledR}$ analysis system (images of microscopic fields abundant in well preserved cells were captured and formatted as .TIFF files, and the immunoexpression of CD3 and CD79 $\alpha$ was calculated). Negative controls were processed in the same manner, using buffer solution instead of primary antibodies. The positive controls for CD3 and CD79 $\alpha$ were cellular samples collected from impression smears of canine hyperplastic lymph nodes. The results of immunocytochemistry were interpreted blindly without knowledge of the lymphoma subtype recognised in routine cytology.

\section{Criteria of lymphoma diagnosis}

The type and subtype of the tumours were determined on the basis of the updated Kiel cytological classification adopted for dogs as previously described (Fournel-Fleury et al., 1997; Fournel-Fleury et al., 2002; Ponce et al., 2010) and supported by the results of immunocytochemistry. For the cytopathological diagnosis the following features were considered: size and shape of cells, volume and intensity of cytoplasm staining, size and shape of nuclei, the position of nucleus in a cell; size, distinctness, number and positioning of nucleoli, and appearance of nuclear chromatin. Additionally, the mitotic rate (MR) was established in cytological specimens according to Fournel-Fleury et al. (1997). Based on the microscopic observation, the diagnosed lymphomas were classified as low-grade (at least $90 \%$ of small cells and low or medium MR) and high-grade lymphomas (at least $80 \%$ of medium and/or large cells and high MR) and, more precisely, as cytological subtypes according to the updated Kiel classification. 


\section{Influence of neutering on lymphoma occurrence}

A case-control study was carried out. Veterinary records from one clinic were browsed for Boxers with and without lymphoma (henceforth referred to as diseased and control group, respectively). Then, their age either at the moment of lymphoma diagnosis (diseased group) or at the moment when they last showed up at the clinic (control group) was recorded, and neutering status was determined by reviewing direct entries in the database, including the description of abdominal surgeries and/or ultrasonographies performed. Then, a control group was built by frequency matching of Boxers without lymphoma at the same age range (Thrusfield, 2007), as the link between age and lymphoma is well known (Grüntzig et al., 2016; Villamil et al., 2009).

\section{Statistical analysis}

Numerical variables were presented as a median, interquartile range (IQR) and range, and compared between groups with a Mann-Whitney $U$ test or a Kruskal-Wallis $\mathrm{H}$ test. Categorical variables were given as a count and percentage, and compared between groups with a Pearson's chi-square test or Fisher's exact test, depending on the expected count of observations in cells of the contingency table. The relationship between neutering and lymphoma was investigated using a Cochran-Mantel-Haenszel test controlling for sex as a potential confounder, since sex appears to be associated with the occurrence of lymphoma (Villamil et al., 2009; Grüntzig et al., 2016). Crude odds ratios (OR) were calculated separately for males and females from partial two-by-two contingency tables and the homogeneity of OR was verified with a Breslow-Day test in Tarone's modification. If the homogeneity of OR proved insignificant, the common OR $\left(\mathrm{OR}_{\mathrm{MH}}\right)$ were calculated with a Mantel-Haenszel procedure. A 95\% confidence interval (CI 95\%) for OR was always reported, and CI 95\% for proportion was calculated using the Wilson score method. The overall survival time (OS) of Boxers with lymphoma was analysed using a Kaplan-Meyer plot. As no censoring was included (all dogs died of lymphoma), OS were compared between groups using a Gehan-Wilcoxon test (2 groups) or generalised Mantel-Cox test (3 groups). The significance level $(\alpha)$ was set at 0.05 and all statistical tests were two-sided. Analysis was performed in Statistica 12 (StatSoft Inc., Tulsa, OK) and IBM SPSS Statistics 24.

\section{Results}

\section{Epidemiology}

During the study period lymphoma was diagnosed in 353 dogs, including 63 Boxers $(17.9 \%$ of all dogs) that met the inclusion criteria. There were no discrepancies between diagnoses of lymphoma and its subtype during the first ex- 
amination and when re-examining the slides, as well as the diagnosis of the subtype of lymphoma and the immunophenotype established by immunocytochemistry. There were 23 males (36.5\%) and 40 females (63.5\%) among the Boxers. Their age ranged from 3 to 14 years with a median of 8 years (IQR from 7 to 10 years; a frequency chart for the age of male and female Boxer dogs is presented in Fig. 1) and did not differ between the sexes $(\mathrm{P}=0.789)$. Age also did not differ between neutered $(n=4)$ and intact $(n=19)$ males $(P=0.806)$, nor between spayed $(\mathrm{n}=22)$ and intact $(\mathrm{n}=18)$ females $(\mathrm{P}=0.320)$.

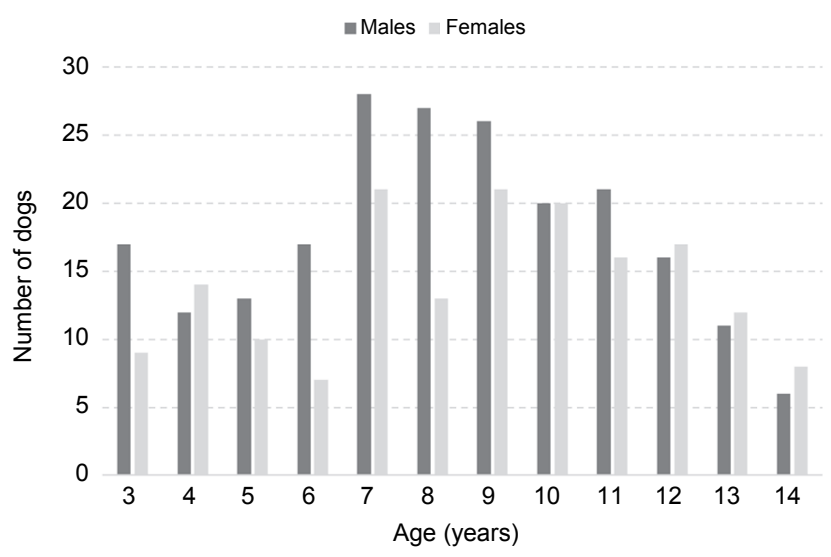

Fig. 1. The number of male and female dogs of different age

\section{Data on neutering}

Data on neutering were available for 63 Boxers with lymphoma and 387 Boxers without lymphoma. As the age of Boxers with lymphoma ranged from 3 to 14 years, a control group was built by matching Boxers without lymphoma at the same age range. This resulted in excluding 64 Boxers younger than 3 years (31 males, 33 females) and 4 Boxers older than 14 years (1 male, 3 females). Eventually, the control group counted 319 Boxers without lymphoma, 145 males $(45.5 \%)$ and 174 females (54.5\%), at the median age of 9 years (IQR from 6 to 11 years), which did not differ from the age of diseased Boxers $(\mathrm{P}=0.586)$. The proportion of castrated individuals in the lymphoma and control group stratified by sex is shown in Fig. 2. Case-to-control ratio was 6.3 in males and 4.4 in females. Female boxers were neutered significantly more often (85 of all 214 females; 39.7\%) than males (26 of all 168 males; $15.5 \%)(\mathrm{P}<0.001)$. This pattern was identical in both the lymphoma and the control groups: in the lymphoma group of Boxers only 4 of 23 males $(17.4 \%$; CI 95\%: 7.0\%, 37.1\%) and as many as 22 of 40 females $(55.0 \%$; CI 95\%: 39.8\%, 69.3\%) were neutered $(\mathrm{P}=0.004)$; in the control group, 22 of 145 males $(15.2 \%$; CI 95\%: 10.2\%, 21.9\%) and 63 of 174 females (36.2\%; CI 95\%: 29.4\%, 43.6\%) were neutered ( $\mathrm{P}<0.001)$. Given 
the expected OR of 2, exposure in controls of $15 \%$ in males and $35 \%$ in females, case-to-control ratio of 6.3 in males and 4.4 in females, and $\alpha=0.05$, the power of this case-control study was only $29 \%$ in males and $51 \%$ in females. For males the OR of neutering was $1.18(0.37,3.79 ; \mathrm{P}=0.785)$. For females the OR of spaying was 2.15 (CI 95\%: 1.07, 4.32; $\mathrm{P}=0.029)$. ORs were homogeneous $(\mathrm{P}=$ 0.383 ) and $\mathrm{OR}_{\mathrm{MH}}$ was 1.84 (CI 95\%: $\left.1.02,3.31 ; \mathrm{P}=0.042\right)$.

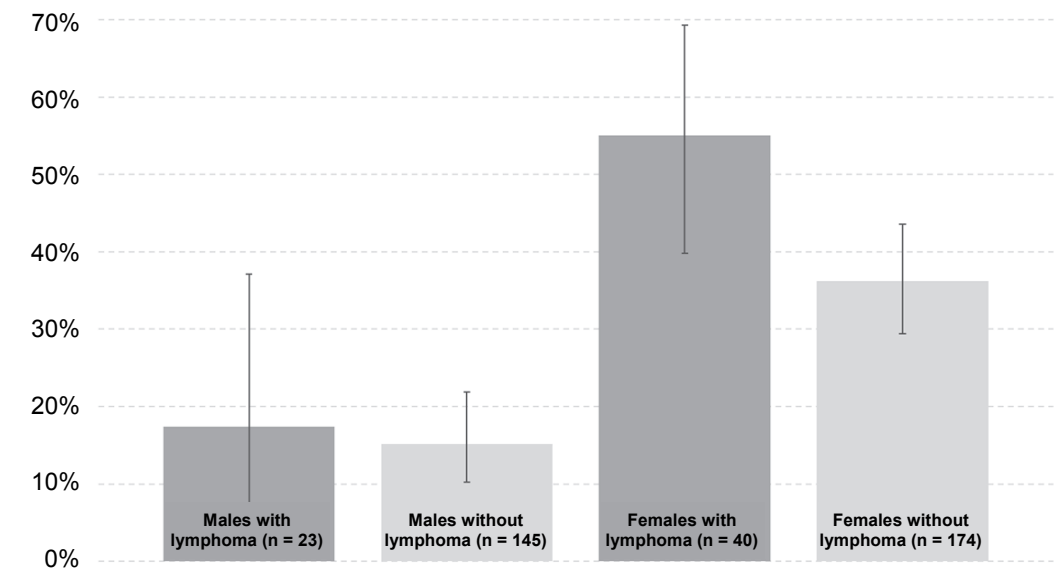

Fig. 2. Proportion of castrated individuals (with a CI of 95\%) in the lymphoma and the control group stratified by sex

Cytology

The immunophenotype was known for 53 (84.1\%) of 63 Boxers with lymphoma; 46 (86.8\%) were T-cell lymphomas and 7 (13.2\%) were B-cell lymphomas. Thirty-four $(64.2 \%)$ were high-grade lymphomas and $19(35.8 \%)$ were lowgrade or indolent lymphomas. According to the Kiel classification the most common subtypes included the pleomorphic, mixed small and large lymphoma (Pleosl; $\mathrm{n}=21 ; 40 \%$ ), clear cell lymphoma (CC; $\mathrm{n}=16 ; 30 \%)$, centroblastic pleomorphic B-cell lymphoma (Cbl; $\mathrm{n}=6 ; 11 \%)$, plasmacytoid high-grade T-cell lymphoma $(\mathrm{n}=3 ; 6 \%)$ and other subtypes $(13 \%)$, including acute lymphoblastic T-cell leukaemia/lymphoma, pleomorphic large cell lymphoma (PleoL), pleomorphic small cell lymphoma (PleoS), prolymphocytic T-cell lymphoma and small lymphocytic/chronic lymphocytic B-cell lymphoma/leukaemia - less than 3 cases of each subtype. According to the WHO classification, among all T-cell lymphomas, 44 (96\% of T-cell lymphomas) were presumably classified as peripheral T-cell lymphomas and 2 (4\% of T-cell lymphomas) as precursor T-cell lymphomas. Among all B-cell lymphomas, 6 were presumably classified as diffuse large B-cell lymphomas and one as small cell B lymphoma. Table 1 summarises the cytological diagnoses of 53 Boxer dogs with lymphoma of confirmed immunophenotype. 
Table 1

Summarised cytological diagnoses for 53 Boxer dogs with lymphomas immunophenotyped by immunocytochemistry

\begin{tabular}{|c|c|c|c|c|}
\hline Types & Grades & WHO classification & Kiel classification & $\begin{array}{l}\text { Number } \\
\text { of cases }\end{array}$ \\
\hline \multirow{7}{*}{ T-cell } & \multirow{5}{*}{ High-grade } & \multirow{4}{*}{$\begin{array}{l}\text { peripheral T-cell } \\
\text { lymphoma }\end{array}$} & $\begin{array}{l}\text { pleomorphic, mixed small and } \\
\text { large lymphoma }\end{array}$ & 21 \\
\hline & & & $\begin{array}{l}\text { plasmacytoid high-grade T-cell } \\
\text { lymphoma }\end{array}$ & 3 \\
\hline & & & pleomorphic large cell lymphoma & 1 \\
\hline & & & prolymphocytic T-cell lymphoma & 1 \\
\hline & & $\begin{array}{l}\text { precursor T-cell } \\
\text { lymphoma }\end{array}$ & $\begin{array}{l}\text { acute lymphoblastic T-cell leukaemia/ } \\
\text { lymphoma }\end{array}$ & 2 \\
\hline & \multirow{2}{*}{ Low-grade } & \multirow{2}{*}{$\begin{array}{l}\text { peripheral T-cell } \\
\text { lymphoma }\end{array}$} & clear cell lymphoma & 16 \\
\hline & & & pleomorphic small cell lymphoma & 2 \\
\hline \multirow{2}{*}{ B-cell } & High-grade & $\begin{array}{l}\text { diffuse large B-cell } \\
\text { lymphoma }\end{array}$ & $\begin{array}{l}\text { centroblastic pleomorphic B-cell } \\
\text { lymphoma }\end{array}$ & 6 \\
\hline & Low-grade & $\begin{array}{l}\text { small cell B lym- } \\
\text { phoma }\end{array}$ & $\begin{array}{l}\text { small lymphocytic/chronic lymphocytic } \\
\text { B-cell lymphoma/leukaemia }\end{array}$ & 1 \\
\hline
\end{tabular}

Boxers with high-grade lymphomas were significantly younger than those with low-grade lymphoma (Fig. 3; $\mathrm{P}=0.036$ ). All Boxers younger than 6 years of age had a high-grade lymphoma. Males and females did not differ with respect to the occurrence of high-grade lymphoma $(\mathrm{P}=0.336)$, nor by lymphoma subtype (pleomorphic, mixed, small and large lymphoma; clear cell lymphoma and centroblastic lymphoma; $\mathrm{P}=0.580$ ).

\section{Clinical findings}

Detailed clinical examination was performed in 29 Boxers with T-cell lymphoma; only 5 of these dogs (17\%) did not have clinical signs (substage a), $24(83 \%)$ dogs manifested clinical signs (substage b). According to the WHO clinical staging, most of the Boxers had clinical stage IV (14 cases, 48\%; involvement of spleen and/or liver) or III (13 cases, 44\%); only one dog had clinical stage I and one dog had clinical stage V ( $4 \%$ of cases; involvement of peripheral blood or/and bone marrow). There were no significant differences in WHO clinical stage Between boxers with low-grade and high-grade lymphomas $(\mathrm{P}=$ 0.322 ), nor were there any differences in the occurrence of various clinical signs (observed by owners or detected at the moment of first presentation) between low-grade and high-grade lymphomas (detailed data not shown). 


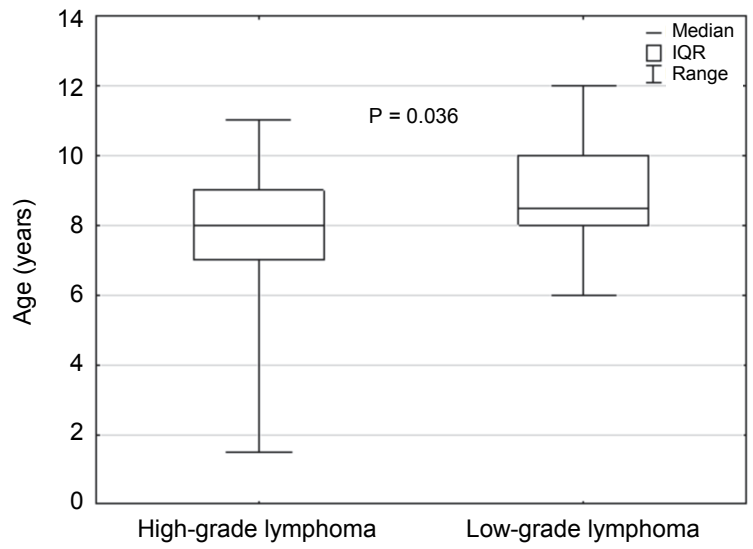

Fig. 3. Comparison of median age of Boxer dogs with high-grade T-cell lymphoma and low-grade T-cell lymphoma. IQR stands for the interquartile range ( $50 \%$ central values)

Table 2

Summarised data on alterations in laboratory parameters in Boxer dogs with lymphoma

\begin{tabular}{l}
\hline Laboratory changes \\
Leukocytosis $(\mathrm{WBC}>12.0 \mathrm{G} / \mathrm{L})$ \\
Anaemia - haematocrit $(\mathrm{HCT}<37 \%)$ \\
Anaemia - haemoglobin $(\mathrm{HGB}<7.45 \mathrm{mmol} / \mathrm{L})$ \\
Anaemia - red blood cell count $(\mathrm{RBC}<5.5 \mathrm{~T} / \mathrm{L})$ \\
Thrombocytopenia $(\mathrm{PLT}<200 \mathrm{G} / \mathrm{L})$ \\
Hypercalcaemia $(\mathrm{Ca}>11.5 \mathrm{mg} / \mathrm{dL})$ \\
Hypoalbuminaemia $(\mathrm{ALB}<33 \mathrm{~g} / \mathrm{L})$ \\
Hyperproteinaemia $(\mathrm{TP}>55 \mathrm{~g} / \mathrm{L})$ \\
Hepatic parameters - alanine aminotransferase $(\mathrm{ALT}>50 \mathrm{U} / \mathrm{L})$ \\
Hepatic parameters - aspartate aminotransferase $(\mathrm{AST}>37 \mathrm{U} / \mathrm{L})$ \\
Hepatic parameters - alkaline phosphatase $(\mathrm{AP}>155 \mathrm{U} / \mathrm{L})$ \\
Hepatic parameters - total bilirubin $(\mathrm{TB}>0.9 \mathrm{mg} / \mathrm{dL})$ \\
Renal parameters - creatinine $(\mathrm{CREA}>1.7 \mathrm{mg} / \mathrm{dL})$ \\
Renal parameters - urea $($ urea $>45 \mathrm{mg} / \mathrm{dL})$
\end{tabular}

Number (\%) of cases

$11(42 \%)$

$3(12 \%)$

$2(8 \%)$

$2(8 \%)$

$11(42 \%)$

$9(36 \%)$

$7(33 \%)$

$3(12 \%)$

$20(80 \%)$

$7(50 \%)$

$4(22 \%)$

$3(20 \%)$

$4(16 \%)$

$6(24 \%)$

\section{Haematology and biochemistry}

There was no association between lymphoma grade and the results of blood analysis, except for total protein concentration, which was significantly lower in Boxers with high-grade lymphoma $(\mathrm{P}=0.031)$. The alterations in laboratory parameters are summarised in Table 2. The leukaemic phase occurred in only one case of high-grade lymphoma (lymphoblastic lymphoma). In the machine marking of blood $109.8 \mathrm{G} / \mathrm{L}$ leukocytes were determined, of which $75 \%$ were regarded as neoplastic lymphocytes. Severe leukocytosis, lymphocytosis and neutropenia were observed in the blood smear, with numerous blastic cells. 
These were large and medium-sized cells. The size of large cells was 2 to 2.5 times larger than the diameter of the red blood cells (RBCs), and the diameter of the medium-sized cells was 1.5 to 1.9 times larger than that of the RBCs. Their nuclei were large, round or with irregular margins. The chromatin pattern was stippled, coarse and the cells had multiple nucleoli.

\section{Survival}

Treatment and/or follow up were analysed for 28 Boxers with T-cell lymphoma (8 dogs with low-grade clear cell lymphoma and 20 dogs with high-grade lymphoma). All dogs died of lymphoma, so no censoring was included in the survival analysis. OS was significantly longer in dogs with low-grade than with high-grade lymphoma - median OS of 6.8 (IQR from 5.1 to 11.6) months and 4.7 (IQR from 2.0 to 6.0 ) months, respectively $(\mathrm{P}=0.024$; Fig. 4). OS was not influenced by WHO clinical stage $(\mathrm{P}=0.805)$, WHO clinical substage $(\mathrm{P}=$ $0.719)$, presence of splenomegaly $(\mathrm{P}=0.864)$, therapy regimen, i.e. chemotherapy vs. glucocorticosteroids vs. no treatment $(\mathrm{P}=0.365$ when dogs with lowgrade and high-grade lymphoma were analysed together or $\mathrm{P}=0.216$ when only dogs with high-grade lymphoma were included; Fig. 5), early administration of glucocorticoids $(\mathrm{P}=0.445)$ or the time from the first presentation to the beginning of chemotherapy $(\mathrm{P}=0.147)$. The median OS for 11 Boxer dogs with highgrade lymphoma without chemotherapy was 2.5 (IQR from 1.5 to 4.8 ) months and was shorter than in 9 Boxer dogs treated with chemotherapy, whose median OS was 4.9 (IQR from 3.5 to 7.1) months, but this difference was not statistically significant $(\mathrm{P}=0.095)$.

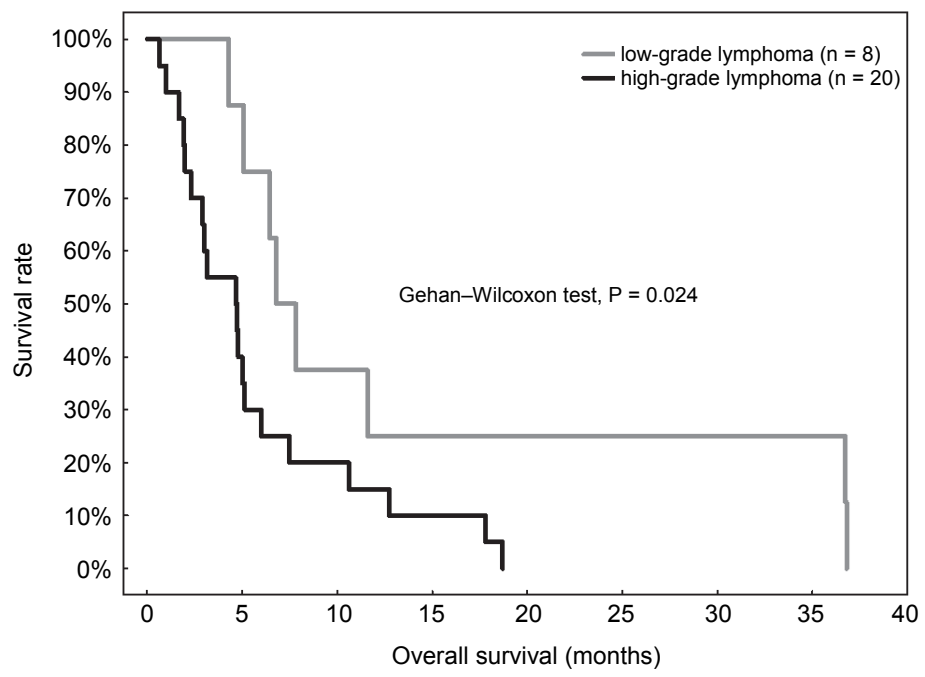

Fig. 4. Kaplan-Meier survival curves in Boxer dogs with low-grade $(\mathrm{n}=8$; median $\mathrm{OS}=6.8$ months $)$ and high-grade lymphomas ( $\mathrm{n}=20$; median $\mathrm{OS}=4.7$ months $)$ 


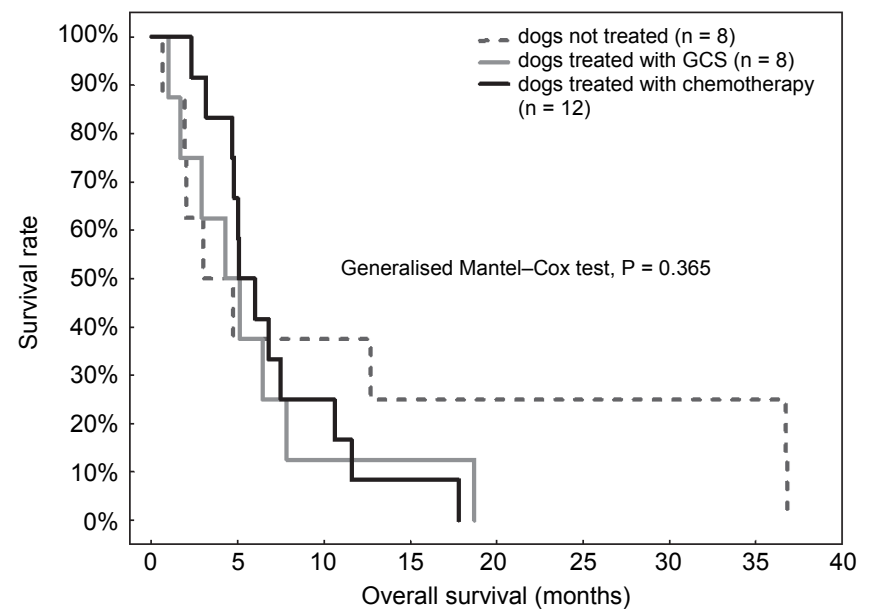

Fig. 5. Kaplan-Meier survival curves in Boxer dogs treated with chemotherapy $(\mathrm{n}=12$; median OS $=5.1$ months), treated with glucocorticosteroids $(\mathrm{n}=8$; median $\mathrm{OS}=4.3$ months $)$ and not treated at all ( $\mathrm{n}=8$; median $\mathrm{OS}=3$ months). $\mathrm{GCS}=$ glucocorticoids

\section{Discussion}

Boxers are a dog breed with a well-known predisposition to lymphoma (Edwards et al., 2004; Jankowska et al., 2015). In American, French, British, and Polish studies, Boxers accounted for $4-13 \%$ of dogs with lymphoma; moreover, Boxers are at a higher risk of T-cell lymphoma tumour development (Lurie et al., 2004; Modiano et al., 2005; Lurie et al., 2008; Pastor et al., 2009; Rebhun et al., 2010; Avery et al., 2014; Jankowska et al., 2015; Fontaine et al., 2017; WilsonRobles et al., 2017). Our study also shows that neutered Boxers are roughly twice more likely to develop lymphoma; however, this applies only to females. Some recently published studies have also found that castration increases the risk of lymphoma in dogs, even though they draw conflicting conclusions regarding the sex group which appears to be predisposed to lymphoma by castration. This sex group are males according to de la Riva et al. (2013), females in our study or both males and females according to Zink et al. (2014). Another study showed that neutered males were in fact at a lower risk of lymphoma development than spayed females (Villamil et al., 2009). Given the low power of our analysis (being a direct consequence of small sample size), our result for males may simply be false negative and should be treated with caution. Therefore, our study appears to add to the general hypothesis that gonadectomised dogs are at a higher risk of lymphoma. Steroid sex hormones have been shown to modulate the function of the immune system (Muñoz-Cruz et al., 2011) and their lack may reduce the ability of the immune system to track and eliminate cells undergoing neoplastic transformation (Engelmann et al., 2011). However, experimental confirmation of this mechanism in dogs is still needed. 
Lymphomas were recognised in Boxers of all ages. In the present study high-grade tumours were diagnosed in younger dogs while low-grade or indolent tumours in older dogs, which is similar to what was shown in a general population of dogs with T-cell lymphoma (Jankowska et al., 2015). Moreover, only high-grade tumours were recognised in dogs younger than 6 years.

Previous reports have suggested that lymphoma in Boxer dogs is a relatively homogeneous disease (Lurie et al., 2004; Lurie et al., 2008). Based on the WHO classification criteria, Lurie et al. (2008) suggested that Boxers could be predisposed to lymphoblastic lymphoma (as many as three fourths of all lymphoma cases occur in this breed). This is at odds with the present study, where lymphoblastic Tcell tumours were recognised less often (only $4.3 \%$ of T-cell lymphomas). Moreover, in other published studies the percentage of lymphoblastic lymphomas classified by the Kiel system and one study based on the NCIWF (National Cancer Institute Working Formulation) in Boxer dogs were closer to our results and comprised $12.5 \%$ and $24-30 \%$ of the cases in a general population of dogs with T-cell lymphomas, respectively (Fournel-Fleury et al., 2002; Ponce et al., 2003). Although there is no clear evidence for the predisposition of Boxers to any specific lymphoma subtype, Pastor et al. (2009) and Avery et al. (2014) have suggested that dogs of this breed can be predisposed to pleomorphic, mixed small and large T-cell tumour - this subtype was also most commonly observed in our study. However, this subtype was rare in a group of Boxer dogs with lymphomas (only $9 \%$ of T-cell lymphomas) analysed by Lurie et al. (2008). Regardless of the classification scheme and the cytomorphologic subtype, among all lymphomas diagnosed in Boxer dogs, high-grade tumours are recognised more often than low-grade lymphomas (Fournel-Fleury et al., 2002; Lurie et al., 2008, Sapierzyński, 2010). Boxers were over-represented in a group of dogs with high-grade T-cell lymphomas but were absent in a group of animals with indolent/low-grade T-zone lymphomas (de la Riva et al., 2013; Mealey et al., 2003). Interestingly, a significant number of Boxers with lymphoma in the present study had clear cell morphology (TZL, Tzone lymphoma according to the WHO classification) and low proliferative activity; this is in contrast to a study conducted by Lurie et al. (2008) and Seelig et al. (2014) where no cases of CC/T zone lymphomas were diagnosed in Boxer dogs.

Like in a general population of dogs with lymphoma, a multicentric form was most often found in Boxers enrolled in our study. Regardless of tumour location and clinical stage of the disease, generally non-specific signs predominated, commonly with polyuria and polydipsia (54\% of all Boxers, including all of the 7 patients with hypercalcaemia). Virtually all dogs were in clinical stage III or IV according to the WHO classification, and most of them were in clinical substage $b$ concerning the occurrence of disease signs, regardless of malignancy grade or cytological subtype; however, significant enlargement of the lymph nodes was observed only in patients with high-grade lymphoma. The administration of glucocorticoids for at least a few weeks before the start of chemotherapy 
could be responsible for the low number of Boxers with marked lymphadenomegaly.

Serious haematological abnormalities were not common in Boxers with lymphoma in the present study, apart from mild leukocytosis and mild thrombocytopenia observed in nearly half of the patients, as well as anaemia that was noted only in dogs with high-grade tumours. Neoplastic lymphocytosis of mild intensity was reported as a common haematological finding in dogs with lowgrade T-zone lymphoma (clear cell lymphoma); however, bone marrow involvement does not seem to be common in such cases (Seelig et al., 2014). Total protein concentration was the only biochemical abnormality with significant differences among low-grade and high-grade tumours, with lower values in the latter lymphoma subtype. Recently a decreased concentration of serum albumin has been found to be related to reduced overall survival and length of remission in dogs with lymphoma (Fontaine et al., 2017).

Treatment and/or follow-up were analysed for 29 Boxers with T-cell lymphoma (9 dogs with low-grade clear cell lymphoma and 20 dogs with high-grade lymphomas). As could be expected, the OS differed between dogs with lowgrade and high-grade T-cell lymphoma. In some cases in the present study prednisone therapy was often the only treatment option; it is well known that an important disadvantage of prednisone treatment is the induction of chemoresistance (Mealey et al., 2003; Schmidt et al., 2004; Gavazza et al., 2009). However, our results did not show a significant effect of prior administration of glucocorticoids on the outcome of treatment with chemotherapy. Median survival time for these patients was 144 days, i.e. 4.8 months. On the other hand, in Boxers with highgrade T-cell lymphomas in the present study, there was little evidence that treatment solely with prednisone was superior to no treatment at all, which corroborates previous observations (Valli et al., 2013).

In the present study the exact treatment protocol slightly varied depending on the clinical course, but the schedule was generally based on CHOP. We believe that the lack of significant differences in median OS in dogs with highgrade tumours treated and not treated with chemotherapy could be, at least partially, explained by the small number of patients in the particular groups. However, it seems that the possibility that high-grade lymphomas in Boxer dogs are clinically more aggressive than in other dog breeds should be considered, so additional studies including larger groups of Boxer dogs with high-grade T-cell lymphoma are warranted. Our results on the dogs' survival are comparable to the findings of Lurie et al. (2008), where all Boxers presented with advanced disease had a median OS of 150 days (the median OS for animals with T-cell tumours was 109 days). Among dogs of all breeds with intermediate or high-grade T-cell lymphomas (lymphoblastic or peripheral T-cell subtypes) from two other studies, the median OS for dogs treated with a CHOP-based protocol was 237 and 235 days, respectively (Rebhun et al., 2010; Avery et al., 2014). In a study published 
by Ponce et al. (2003), the median OS for pleomorphic mixed T-cell and lymphoblastic lymphomas was 14 and 9 months, respectively. Our findings and the results of Lurie et al. (2008) may suggest that Boxer dogs may respond less favourably to chemotherapy than patients of other breeds in which outcomes can be longer (Rebhun et al., 2010; Avery et al., 2014). On the other hand, in a study reported by Valli et al. (2013), the median OS for dogs with high-grade T-cell lymphomas was 162 days. However, in this study lymphomas were divided into groups according to the degree of malignancy (high grade, intermediate grade and low grade). The four most common breeds of large dogs (75.6\% of cases) in descending order were Golden Retrievers, Labrador Retrievers, crossbreed dogs and Boxers, so the number of Boxers could influence the outcome of treatment.

Among all dogs with various subtypes of lymphoma, animals with lowgrade T-cell (T-zone) tumours have the longest median OS (Ponce et al., 2003; Valli et al., 2006; Flood-Knapik et al., 2012; Frantz et al., 2013; Valli et al., 2013). Similarly, OS was the longest in Boxers with clear cell lymphoma (Tzone lymphoma) in the present study; however, the median OS was not as long as in the studies mentioned above (from 21 to 33.5 months vs. 6.8 months in our study). Moreover, there was a significant difference in OS between dogs receiving multi-drug chemotherapy when compared to patients receiving solely prednisone, and dogs without treatment in the study of Valli et al. (2006). In another study the outcomes in dogs with T-zone lymphoma were longest in patients treated without an aggressive chemotherapy schedule (Flood-Knapik et al., 2012). In our study the longest survival was achieved in dogs with CC lymphoma without treatment (only 2 dogs, survival time of 1104 and 1802 days). Another three Boxers with low-grade clear cell lymphoma had stage III disease, but they had clinical signs (substage IIIb), so chemotherapy with multi-agent protocol was introduced. Unfortunately, the OS was relatively short in these cases (median of 204 days) and similar to the median OS for patients treated with prednisolone alone. Dogs with T-zone/CC lymphoma in the present study were probably diagnosed during later stages of the disease, as clinical signs associated with lymphoma spread were rarely present. Therefore, it should be taken into account that the diagnosis was made in the late stage of the disease, hence OS were shorter than expected. According to Aresu et al. (2013), the prognosis of advanced indolent lymphoma does not appear to be appreciably different from that of highgrade tumours.

The main disadvantage of our study is the relatively small group of Boxers with complete clinical data and the heterogeneous chemotherapy schedules that were used. The Boxer dogs included in the analysis of treatment were collected only from a referral centre, and commonly were referred by general practitioners, thus most of them had an advanced form of the disease $(83 \%$ of patients were in substage B). So, it is possible that differences between OS of Boxers in the present study and those presented by other authors (Ponce et al., 2003; Rebhun et 
al., 2010; Avery et al., 2014) may result from the small number of patients with a given lymphoma chemotherapy schedule and disease in the advanced stage. The second limitation of the study is the lack of histopathological confirmation of lymphoma diagnosis. However, many authors have recommended cytology as an adequate method for canine lymphoma diagnosis, especially when supported by additional tests that allow for immunophenotype confirmation of neoplastic cells (immunocytochemistry and/or flow cytometry). Recently, in at least three studies focused on canine lymphomas, cytological diagnosis has been one of the inclusion criteria (Brown et al., 2017; Fontaine et al., 2017; Wilson-Robles et al., 2017). Fortunately, the cytological picture of most common subtypes of canine lymphoma is highly specific.

In the present study no detailed analysis of the immunophenotype of neoplastic cells was performed. This parameter has recently been shown to have a significant impact on the efficacy of treatment in dogs with T-cell lymphoma. Avery et al. (2014) and Seelig et al. (2014) have proved that the immunoexpression of CD45 significantly correlates with the biologic behaviour of canine TCL lymphoma. More recently, Deravi et al. (2018) have shown that treatment outcomes can be predicted by immunophenotype in respect of CD4, CD8, and MHCII immunoexpression. Unfortunately, these antibodies were not available in the present study, and the patients had been collected between 2009 and 2015.

Concluding, Boxer dogs are predisposed to T-cell lymphoma, with predominance of high-grade tumour, especially pleomorphic, mixed small and large T-cell subtype. Neutered Boxers are roughly twice more likely to develop lymphoma; however, this applies only to females. There are no significant differences in clinical and laboratory parameters between low-grade and high-grade lymphomas. It is possible that Boxer dogs may respond less favourably to chemotherapy than patients of other breeds.

\section{References}

Aresu, L., Martini, V., Rossi, F., Vignoli, M., Sampaolo, M., Aricò, A., Laganga, P., Pierini, A., Frayssinet, P., Mantovani, R. and Marconato, L. (2013): Canine indolent and aggressive lymphoma: clinical spectrum with histologic correlation. Vet. Comp. Oncol. 13, 348-362.

Avery, P. R., Burton, J., Bromberek, J. L., Seelig, D. M., Elmslie, R., Correa, S., Ehrhart, E. J., Morley, P. S. and Avery, A. C. (2014): Flow cytometric characterization and clinical outcome of CD4+ T-cell lymphoma in dogs: 67 causes. J. Vet. Intern. Med. 28, 538-546.

Brown, P. M., Tzannes, S., Nguyen, S., White, J. and Langova, V. (2017): LOOP chemotherapy as a first-line treatment for dogs with T-cell lymphoma. Vet. Comp. Oncol. 16, 108-113.

Cadieu, E. and Ostrander, E. A. (2007): Canine genetics offers new mechanisms for the study of human cancer. Cancer Epidemiol. Biomarkers Prev. 16, 2181-2183.

Caniatti, M., Roccabianca, P., Scanziani, E., Paltrinieri, S. and Moore, P. F. (1996): Canine lymphoma: immunocytochemical analysis of fine-needle aspiration biopsy. Vet. Pathol. 33, 204-212. 
de la Riva G. T., Hart, B. L., Farver, T. B., Oberbauer, A. M., Messam, L. L., Willits, N. and Hart, L. A. (2013): Neutering dogs: effects on joint disorders and cancers in Golden Retrievers. PLos One 8, e55937.

Deravi, N., Berke, O., Woods, J. P. and Bienzle, D. (2017): Specific immunophenotypes of canine $\mathrm{T}$ cell lymphoma are associated with different outcomes. Vet. Immunol. Immunopathol. 191, 5-13.

Dobson, J. M. (2013): Breed-predispositions to cancer in pedigree dogs. ISRN Vet. Sci. 2013, 1-11.

Edwards, D. S., Henley, W. E., Harding, E. F., Dobson, J. M. and Wood, J. L. N. (2004): Breed incidence of lymphoma in a UK population of insured dogs. Vet. Comp. Oncol. 4, 200-206.

Engelmann, F., Barron, A., Urbanski, H., Neuringer, M., Kohama, S. G., Park, B. and Messaoudi, I. (2011): Accelerated immune senescence and reduced response to vaccination in ovariectomized female rhesus macaques. AGE 33, 275-289.

Flood-Knapik, K. E., Durham, A. C., Gregor, T. P., Sanchez, M. D., Durney, M. E. and Sorenmo, K. U. (2012): Clinical, histopathological and immunohistochemical characterization of canine indolent lymphoma. Vet. Comp. Oncol. 11, 272-286.

Fontaine, S. J., McCulloch, E., Eckersall, P. D., Haining, H., Patterson Kane, J. C. and Morris, J. S. (2017): Evaluation of the modified Glasgow Prognostic Score to predict outcome in dogs with newly diagnosed lymphoma. Vet. Comp. Oncol. 15, 1513-1526.

Fournel-Fleury, C., Magnol, J. P., Bricaire, P., Marchal, T., Chabanne, L., Delverdier, A., Bryon, P. A. and Felman, P. (1997): Cytohistological and immunological classification of canine malignant lymphomas: comparison with human non Hodgkin's lymphomas. J. Comp. Pathol. 117, 35-59.

Fournel-Fleury, C., Ponce, F., Felman, P., Blavier, A., Bonnefont, C., Chabanne, L., Marchal, T., Cadore, J. L., Goy-Thollot, I., Ledieu, D., Ghernati, I. and Magnol, J. P. (2002): Canine Tcell lymphomas: a morphological, immunological, and clinical study of 46 new cases. Vet. Pathol. 39, 92-109.

Frantz, A. M., Sarver, A. L., Ito, D., Phang, T. L., Karimpour-Fard, A., Scott, M. C., Valli, V. E., Lindblad-Toh, K., Burgess, K. E., Husbands, B. D., Henson, M. S., Borgatti, A., Kisseberth, W. C., Hunter, L. E., Breen, M., O’Brien, T. D. and Modiano, J. F. (2013): Molecular profiling reveals prognostically significant subtypes of canine lymphoma. Vet. Pathol. 50, 693-703.

Garrett, L. D., Thamm, D. H., Chun, R., Dudley, R. and Vail, D. M. (2002): Evaluation of a 6month chemotherapy protocol with no maintenance therapy for dogs with lymphoma. J. Vet. Intern. Med. 16, 704-709.

Gavazza, A., Sacchini, F., Lubas, G., Gugliucci, B. and Valori, E. (2009): Clinical, laboratory, diagnostic and prognostic aspects of canine lymphoma: a retrospective study. Comp. Clin. Path. 18, 291-299.

Grüntzig, K., Graf, R., Boo, G., Guscetti, F., Hässig, M., Axhausen, K. W., Fabrikant, S., Welle, M., Meier, D., Folkers, G. and Pospischil, A. (2016): Swiss Canine Cancer Registry 19552008: Occurrence of the most common tumour diagnoses and influence of age, breed, body size, sex and neutering status on tumour development. J. Comp. Pathol. 155, 156-170.

Jankowska, U., Jagielski, D., Czopowicz, M. and Sapierzyński, R. (2015): The animal-dependent risk factors in canine T-cell lymphomas. Vet. Comp. Oncol. 15, 307-314.

Lurie, D. M., Lucroy, M. D., Griffey, S. M., Simonson, E. and Madewell, B. R. (2004): T-cell derived malignant lymphoma in the Boxer breed. Vet. Comp. Oncol. 2, 171-175.

Lurie, D. M., Milner, R. J., Suter, S. E. and Vernau, W. (2008): Immunophenotypic and cytomorphologic subclassification of T-cell lymphoma in the Boxer breed. Vet. Immunol. Immunopathol. 125, 102-101.

Mealey, K. L., Bentjen, S. A., Gay, J. M. and Hosick, H. L. (2003): Dexamethasone treatment of a canine, but not human, tumour cell line increases chemoresistance independent of Pglycoprotein and multidrug resistance-related protein expression. Vet. Comp. Oncol. 1, 67-75. 
Modiano, J. F., Breen, M., Burnett, R. C., Parker, H. G., Inusah, S., Thomas, R., Avery, P. R., Lindblad-Toh, K., Ostrander, E. A., Cutter, G. C. and Avery, A. C. (2005): Distinct B-cell and T-cell lymphoproliferative disease prevalence among dog breeds indicates heritable risk. Cancer Res. 65, 5654-5661.

Muñoz-Cruz, S., Togno-Pierce, C. and Morales-Montor, J. (2011): Non-reproductive effects of sex steroids: their immunoregulatory role. Curr. Top. Med. Chem. 11, 1714-1727.

Olson, P. N. (2007): Using the canine genome to cure cancer and other diseases. Theriogenology 68, 378-381.

Pastor, M., Chalvet-Monfray, K., Marchal, T., Keck, G., Magnol, J. P., Fournel-Fleury, C. and Ponce, F. (2009): Genetic and environmental risk indicators in canine non-Hodgkin's lymphomas: breed association and geographic distribution of 608 cases diagnosed throughout France over 1 year. J. Vet. Intern. Med. 23, 301-310.

Ponce, F., Magnol, J. P., Marchal, T., Chabanne, L., Ledieu, D., Bonnefont, C., Felman, P. and Fournel-Fleury, C. (2003): High-grade canine T-cell lymphoma/leukemia with plasmacytoid morphology: a clinical pathological study of nine cases. J. Vet. Diagn. Invest. 15, 330-337.

Ponce, F., Marchal, T., Magnol, J. P., Turinelli, V., Ledieu, D., Bonnefont, C., Pastor, M., Delignette, M. L. and Fournel-Fleury, C. (2010): A morphological study of 608 cases of canine malignant lymphoma in France with a focus on comparative similarities between canine and human lymphoma morphology. Vet. Pathol. 47, 414-433.

Rebhun, R. B., Kent, M. S., Borrofka, S. A. E. B., Frazier, S., Skorupski, K. and Rodriguez, C. O. (2010): CHOP chemotherapy for the treatment of canine multicentric T-cell lymphoma. Vet. Comp. Oncol. 9, 38-44.

Ruslander, D. A., Gebhard, D. H. and Tompkins, M. B. (1997): Immunophenotypic characterization of canine lymphoproliferative disorders. In Vivo 11, 169-172.

Sapierzyński, R. (2010): Practical aspects of immunocytochemistry in canine lymphomas. Pol. J. Vet. Sci. 13, 661-668.

Schmidt, S., Rainer, J., Ploner, C., Presul, E., Riml, S. and Kofler, R. (2004): Glucocorticoidinduced apoptosis and glucocorticoid resistance: molecular mechanisms and clinical relevance. Cell Death Differ. 11, 45-55.

Seelig, D. M., Avery, P., Webb, T., Yoshimoto, J., Bromberek, J., Ehrhart, E. J. and Avery, A. C. (2014): Canine T-zone lymphoma: unique immunophenotypic features, outcome, and population characteristics. J. Vet. Intern. Med. 28, 878-886.

Shearin, A. L. and Ostrander, E. A. (2010): Leading the way: canine models of genomics and disease. Dis. Model Mech. 3, 27-34.

Sutter, N. B. and Ostrander, E. A. (2004): Dog star rising: the canine genetic system. Nat. Rev. Genet. 12, 900-910.

Thrusfield, M. (2007): Veterinary Epidemiology. 3rd Edition, Blackwell Science Ltd., Oxford.

Valli, V. E., Kass, P. H., San Myint, M. and Scott, F. (2013): Canine lymphomas: association of classification type, disease stage, tumor subtype, mitotic rate, and treatment with survival. Vet. Pathol. 50, 738-748.

Valli, V. E., Vernau, W., de Lorimier, L. P., Graham, P. S. and Moore, P. F. (2006): Canine indolent nodular lymphoma. Vet. Pathol. 43, 241-256.

Villamil, J. A., Henry, C. J., Hahn, A. W., Bryan, J. N., Tyler, J. W. and Caldwell, C. W. (2009): Hormonal and sex impact on the epidemiology of canine lymphoma. J. Cancer Epidemiol. 2009, 591753.

Wilson-Robles, H., Budke, C. M., Miller, T., Dervisis, N., Novosad, A. and Wright, Z. (2017): Geografical differences in survival of dogs with non-Hodgkin lymphoma treated with CHOP based chemotherapy protocol. Vet. Comp. Oncol. 15, 1564-1571.

Zink, M. C., Farhoody, P., Elser, S. E., Ruffini, L. D., Gibbons, T. A. and Rieger, R. H. (2014): Evaluation of the risk and age of onset of cancer and behavioral disorders in gonadectomized Vizslas. J. Am. Vet. Med. Assoc. 244, 309-319. 